\title{
Formation of a smectic A phase from a strongly deformed planar nematic liquid crystal CBOOA (*)
}

\author{
H. P. Hinov $\left({ }^{* *}\right)$ \\ Laboratoire de Physique des Solides, Bât. 510, Université de Paris-Sud, 91405 Orsay, France
}

(Reçu le 22 août 1979, révisé le 8 septembre 1980, accepté le 14 octobre 1980)

\begin{abstract}
Résumé. - On refroidit une couche nématique planaire déformée de CBOOA pour passer en phase smectique A. Cette phase apparaît d'abord au milieu de la cellule, là où les molécules sont parallèles au champ électrique appliqué. L'orientation des couches smectiques près des surfaces dépend de la force du champ électrique, de la vitesse du refroidissement et de la force d'ancrage des molécules aux surfaces limites.

Pour des champs électriques très élevés, l'adaptation`aux conditions d'ancrage se fait par des domaines confocaux dans lesquels l'ellipse est dégénérée en un cercle et l'hyperbole en une droite. Le remplissage de l'espace est plus aléatoire qu'Appolonien et s'arrête pour des domaines confocaux dont le rayon critique dépend de la force d'ancrage. On calcule l'énergie d'ancrage.
\end{abstract}

\begin{abstract}
The SmA phase is obtained after cooling of a strongly deformed planar nematic CBOOA. This phase first appears in the middle of the liquid crystal cell where the molecules are parallel to the applied electric field. The orientation of the smectic layers in the boundary regions depends on the strength of the electric field, on the rate of cooling and on the strength of the surface anchoring.

At higher electric fields matching to the boundary conditions is made with a system of confocal domains with ellipses degenerated into circles and hyperbolae degenerated into straight lines. Filling of space is mainly random rather than Appolonian and stops when confocal domains have some critical circular radius which depends on the strength of the surface coupling. The surface energies in the smectic and nematic phases are also calculated.
\end{abstract}

1. Introduction. - The nematic to smectic A phase transition has been much studied after the work of de Gennes [1] and McMillan [2] who introduced a phenomenological Landau theory to describe the static deformations of the smectic order parameter $\psi$ and the nematic director $\mathbf{n}$. This work extented the Oseen-Frank theory of the SmA phase to describe the static behaviour of the nematic phase just above the phase transition where cybotactic groups exist. Different correlation lengths $\sim\left(T-T_{\mathrm{c}}\right)^{-1 / 2}$ or $\sim\left(T-T_{\mathrm{c}}\right)^{-2 / 3}$ were calculated according to the different versions of the classical Landau theory used, namely mean field theory (McMillan) and liquid helium analog (de Gennes) respectively. Further progress was made by Brochard [3] who postulated a dynamic scaling relation for fluctuations of the director $\delta$ n and by Jähning and Brochard [4] who improved the modelling of the viscosity divergence and of the elastic coefficients of the nematics. Halperin

(*) Part of this paper was presented at Eight Int. Liq. Cryst. Conf., Kyoto, Japan, June 30-July 4, 1980, Code No. J-1P.

(**) Permanent address : Institute of Solid State Physics, Bulgarian Academy of Sciences, Sofia 1184, Bulgaria. and Lubenski [5], [6] changed de Gennes' version to include a transformed order parameter in the smectic phase which does not have divergent phase fluctuations (N-SmA-slightly first order). The divergence of the nematic elastic constants was also calculated by Jing-Huei Chen and Lubensky [7] and the divergence of the viscosity coefficients by McMillan [8], Martins et al. [9] and Hossain et al. [10]. Experiments performed include X-ray scattering [11]-[13], elastic coherent neutron scattering [14] and light scattering [15]-[21]. NMR measurements [22], acoustic measurements [23], Fredericks transition measurements [24]-[28] and other methods [29]-[32] have clearly showon that in the vicinity of the N-SmA phase transition there is a strong divergence of the twist and bend elastic coefficients, the rotational viscosity coefficient $\gamma_{1}$ and some other viscosity coefficients. The divergence of the elastic coefficient $\delta K_{33}$ is more drastic than that of the twist elastic coefficient $\delta K_{22}$. At and below the N-SmA phase transition their ratio is $\delta K_{33} / \delta K_{22} \sim 8$. There is no divergent anomaly in the elastic coefficient $K_{11}$ with only one exception [27]. These results show that the smectic clusters in the nematic phase appear as a rotational ellipsoid which has its long axis perpen- 
dicular to the layers. On approaching $T_{\mathrm{c}}$ the length of this axis corresponding to the number of smectic layers increases critically, whereas the lateral extension of the layers increases more slowly. Consequently the critical fluctuations driving the formation of the smectic clusters are those which lead to a stacking of the layers. This result is relevant to the formation of the SmA phase from deformed nematics.

In this paper we describe the SmA phase obtained experimentally by cooling from different a.c. electrically deformed planar nematic liquid crystals CBOOA (cyanobenzylidene-octyloxyaniline). The textures of the SmA phase depends strongly on the initial elastic state of the nematic layer (the value of the bendsplay deformations) and on the temperature in the smectic phase.

At voltages $U \leqslant 1.5 U_{\text {th }}\left(U_{\text {th }}\right.$ is the threshold voltage for the deformation in the nematic layer) the SmA phase grows in the form of the well known bend walls (or Grandjean's walls) [34], [35] etc.

At voltages between $1.5 U_{\text {th }}$ and $3 U_{\text {th }}$ the SmA textures observed confirmed the experimental results of Cladis and Torza [35] for the formation of SmA phase from an initially bent nematic layer with homeotropic orientation at one surface and planar orientation at the other surface. The virgule and honeycomb textures observed in the SmA phase clearly show that nucleation of the smectic planes starts at the boundaries where the temperature is lower and propagates to the middle of the liquid crystal layer.

At higher voltages we observed grain boundaries as well as different types of confocal domains depending on the value of the temperature in the smectic phase, on the coherence length in the nematic phase and on the surface anchoring. These SmA textures are a result of the different orientations of the liquid crystal molecules at the surfaces and in the bulk of the layer.

The grain boundaries have been widely studied both theoretically [36]-[38] and experimentally [38]-[41].

The confocal domains observed in our experiment were usually in the form of ellipses degenerated into circles and hyperbolae degenerated into straight lines (Rosenblath et al. [42] pointed out a number of difficulties with their formation in initially planar SmA layers). Toroidal confocal domains built in one homeotropic SmA matrix have been obtained by : (a) Perez and Proust [43] for $8 \mathrm{CB}$ confined between two solid surfaces treated with one molecular layer of an alkylpolyvenyl pyridine bromide; namely $\mathrm{PVPC}_{4}$ and PVPC $_{16}$ to ensure planar and homeotropic orientation respectively and (b) Le Berre and Hareng [44], [45] after relaxation of homeotropic SmA CBOOA in the presence of an electric field near the N-SmA phase transition. However these authors did not consider this particularly important case which allows a simple determination of the value of the surface coupling parameter $W_{\mathrm{s}}$. The interpretation [46] of our results is easier because our experiment is simpler than previous ones.

The formation of these adjusted structures shows that the SmA phase starts to nucleate either all at once in the entire liquid crystal layer $\left(U \leqslant 5 U_{\text {th }}\right)$ or first in the middle of the liquid crystal layer. The latter is a more likely process $\left(U \geqslant 5 U_{\text {th }}\right)$. This idea is justified by the value of $T_{\mathrm{c}}$ (the phase transition temperature N-SmA) which strongly decreases with increase of elastic deformations in the nematic phase [35]. The adjustment process is in agreement with Bouligand's idea [37] but with two exceptions : (i) filling of the space is frequently random and only, in rare cases Appolonian, and (ii) stops for circular confocal domains having some critical radius which depends on the strength of the surface coupling. Further filling of the space is with homeotropic SmA with planes parallel to the glass plates. However, this is exactly the picture, proposed by Friedel [47] for matching of the bulk orientation with the free surface orientation of a SmA liquid crystal drop supported by one solid substrate.

The formation of circular-line confocal domains allows for a simple determination of the surface coupling parameter, $W_{\mathrm{s}}$, in the SmA phase. At very high electric fields $\left(\left(U>15 U_{\mathrm{th}}\right), d=26 \mu \mathrm{m}\right)$ the liquid crystal molecules in the nematic and smectic A phase were homeotropic, which allows for a simple determination of the surface coupling parameter $W_{\mathbf{s N}}$ in the nematic phase.

2. Experiments and experimental results. - 2.1 Preparation OF THE CELls. - The liquid crystal under investigation, CBOOA, sandwiched between two nesa coated glass plates, separated by $26 \mu \mathrm{m}$ mylar spacers, was placed between two lateral metal holders (Fig. 1). The mechanical stability was achieved by means of four screws. The cell was first warmed with a regulated electric heater. The sample was placed on the stage of a Leitz microscope when the liquid crystal had reached its isotropic phase. The massive metal lateral holders stored thermal energy such that the cooling rate was $15^{\circ} \mathrm{C} / \mathrm{min}$., sufficient for the performance of the experiment. The preparation of the liquid crystal cells in this way has several advantages, namely, the possibility of achieving high temperature gradients in the electrode planes, easy investigation of a large area (several $\mathrm{cm}^{2}$ ) of the liquid crystal and photography of the liquid crystal structures with

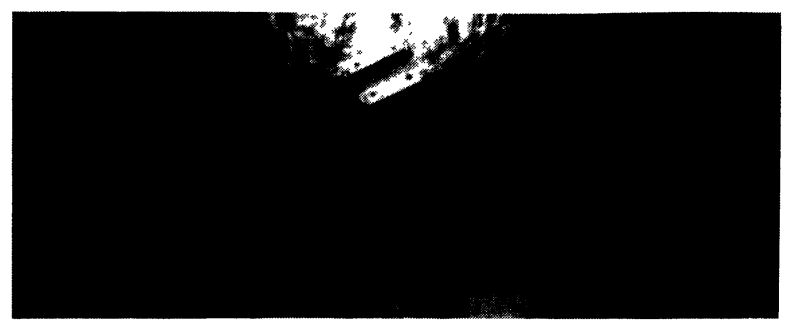

Fig. 1. - Structure of the liquid crystal cell with two glass plates. 
objectives having small focal lengths (magnification $\times 400$ ). The experimental results were repeated using a Mettler hot stage with temperature regulation rates $0.1^{\circ} \mathrm{C} / \mathrm{min}$., $1^{\circ} \mathrm{C} / \mathrm{min}$., $3^{\circ} \mathrm{C} / \mathrm{min}$. and $10^{\circ} \mathrm{C} / \mathrm{min}$. The phase transition temperatures were :

$$
\mathrm{I}_{109^{\circ} \mathrm{C}} \mathrm{N}_{83.0{ }^{\circ} \mathrm{C}} \mathrm{SmA}{ }_{73.0{ }^{\circ} \mathrm{C}} \mathrm{C} \text {. }
$$

Planar orientation of the nematic liquid crystal was obtained by $\mathrm{SiO}$ treatment of the electrodes under vacuum evaporation. A high-frequency $(>20 \mathrm{kHz})$ voltage source of magnitude between $\sim 1.1 U_{\text {th }}$ and $40 U_{\text {th }}\left(U_{\text {th }}\right.$ is the threshold voltage for the deformations in the nematic phase) was applied to the liquid crystal layer in the nematic phase. Formation of the SmA phase was different and depended on both the coherence length $\xi$ introduced by de Gennes for the nematic phase and on the cooling rate.

2. 2 Thermal Distribution IN THE CELlS. - Thermal transport phenomena in the liquid crystals have been insufficiently investigated theoretically and experimentally [48]. Earlier investigations showed a strong dependence of the thermal conductivity on temperature, liquid-crystal orientations and the temperature gradients. The thermal conductivity at the bounding surfaces is usually greater [49], [50] than that in the bulk of the liquid crystal layer. Recent investigations by Urback et al. [51] and by Rondelez et al. [52] clearly show a positive sign of the thermal conductivity anisotropy, i.e. the thermal conductivity along the molecules is always higher than the thermal conductivity along the perpendicular direction. There is no dramatic change around the transition N-SmA or N-SmC i.e. only short-range geometrical factors are important.

Cooling of the cells investigated clearly results in a high temperature gradient in the electrode planes : from the lateral metal holders storing the thermal energy to the middle part of the cells.

However, it is difficult to understand the temperature distribution along $Z$ (normal to the glass plates). A temperature gradient is expected because of the difference in the thermal properties of the glass on the microscopic stage and the air above the liquid crystal cell. This asymmetry, however, is not significant because of the massive lateral metal holders with an homogeneous temperature distribution. On the other hand the cell thickness $(20-30 \mu \mathrm{m})$ is much smaller than that $(\sim 1 \mathrm{~cm})$ of the metal holders. This was confirmed in our experiment by placing one complementary glass plate above the cells. No changes in the experimental results were obtained.

Our experiments show that the thermal gradient along $Z$ decreases with thickness of the liquid crystal layer and is smaller with $10 \mu \mathrm{m}$ thick cells.

2.3 MicROSCOPIC OBSERVATIONS. - In the slightly deformed nematic liquid crystal the SmA phase starts to nucleate at the boundaries where in agreement with Cladis and Torza's results [35] the nucleation energy is zero (Fig. 2). Such nucleation processes determine the formation of stable grain boundaries (typical regions are marked as A in Fig. 3) already observed by Goscianski et al. [40] in homeotropic CBOOA SmA, in the presence of a deforming electric field. Usually the various grain boundaries are unstable and relax to focal conics. The stability of the grain boundary nucleation process has been investigated in detail by Cladis and Torza [35].

Further increase of the voltage $\left(U \sim 5 U_{\mathrm{th}}\right)$ in the nematic phase leads to generation of typical focal conics in the layers (Fig. 2). Careful scrutiny of the patterns given in figures 4 and 5 should reveal the following types of confocal domains :

a) The general case of Dupin cyclides [33], [39], [41], [47], [53], [54] in the section parallel to the hyperbola

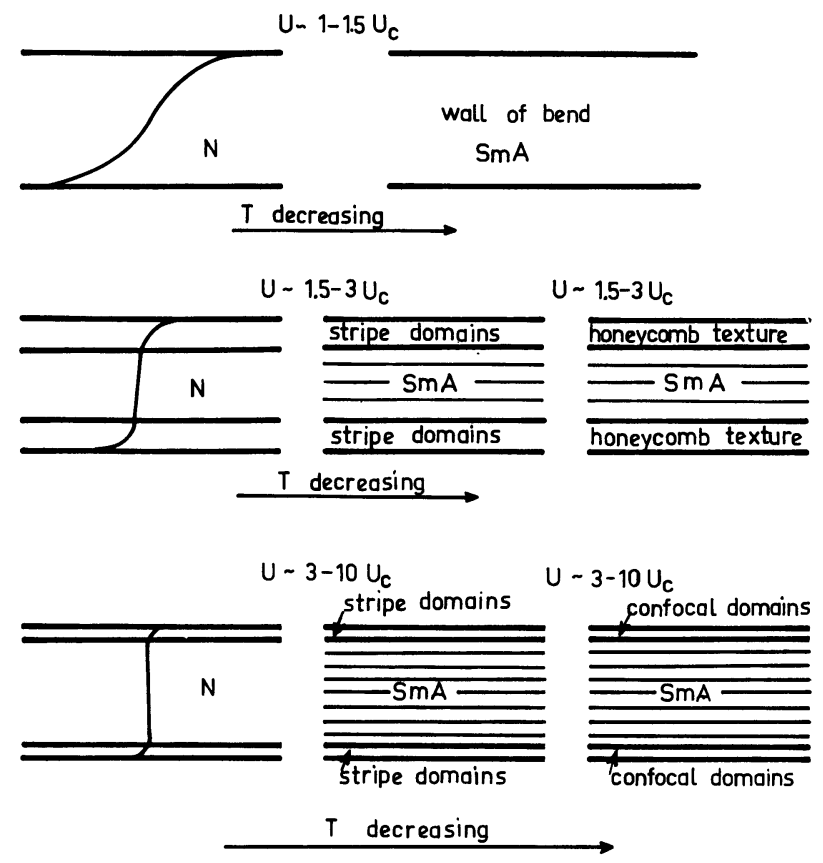

Fig. 2. - Various textures obtained in planar CBOOA in the deformed nematic phase.

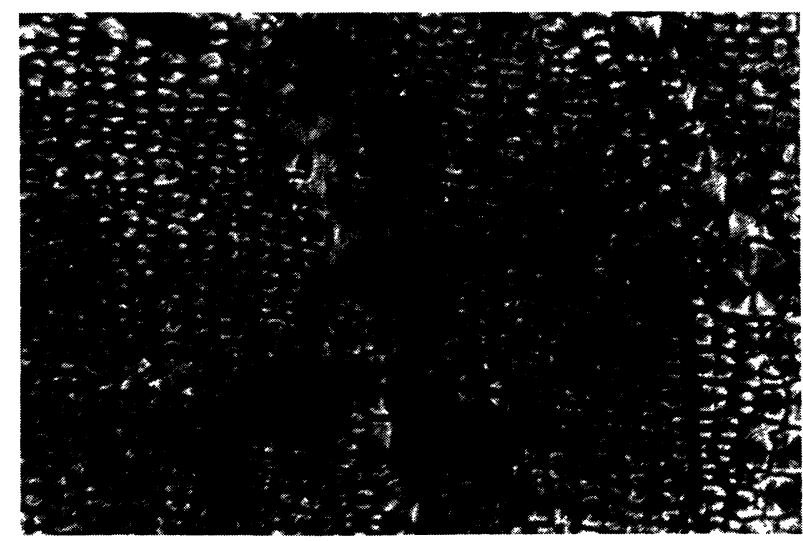

Fig. 3. - Grain boundaries in planar CBOOA $(26 \mu \mathrm{m}$ thick, $U=3 \mathrm{~V}, n_{0} / / \mathrm{P} \perp \mathrm{A}$, cooling rate $15^{\circ} \mathrm{C} / \mathrm{min}$.). Typical grain boundaries are designated by $\mathrm{A}$. The short side of the photo corresponds to $215 \mu \mathrm{m}$ (magnif. $\times 320$ ). 


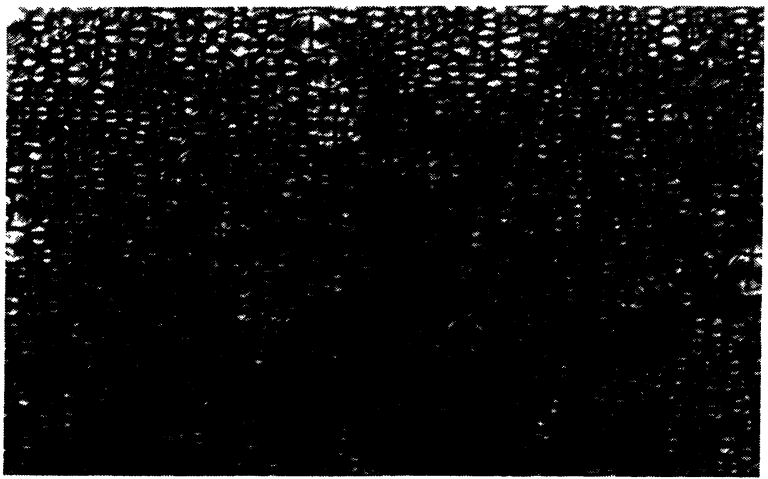

Fig. 4. - General conic structures in planar CBOOA ( $26 \mu \mathrm{m}$ thick, $U=5 \mathrm{~V}, n_{0} / / \mathrm{P} \perp \mathrm{A}$, cooling rate $15^{\circ} \mathrm{C} / \mathrm{min}$.). The general case of Dupin cyclides in the section parallel to the hyperbola plane is designated by $\mathbf{B}$, and the general case of Dupin cyclides in the section parallel to the ellipse plane by $\mathrm{C}$. The short side of the photo corresponds to $215 \mu \mathrm{m}$ (magnif. $\times 320$ ).

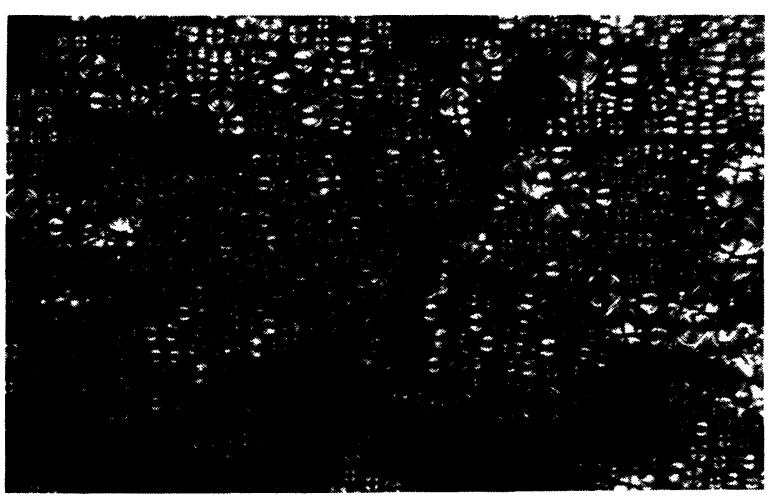

Fig. 5. - General conic structures in planar CBOOA $(26 \mu \mathrm{m}$ thick, $U=5 \mathrm{~V}, n_{0} / / \mathrm{P} \perp \mathrm{A}$, cooling rate $15^{\circ} \mathrm{C} / \mathrm{min}$.). The general case of Dupin cyclides in the section parallel to the hyperbola plane is designated by B, the general case of Dupin cyclides in the section parallel to the ellipse plane by C. Single confocal domains, built in a homeotropic SmA matrix in which the ellipses are degenerated into circles and the hyperbolae into straight lines by $\mathrm{D}$ and homeotropic SmA regions with planes parallel to the glass plates by $\mathrm{E}$. The short side of the photo corresponds to $215 \mu \mathrm{m}$ (magnif. $\times 320$ ).

plane [55] (designated by B in the photos) or in the section parallel to the ellipse plane [55] (designated by $\mathrm{C}$ ).

b) Single confocal domains, built in a homeotropic SmA matrix, in which the ellipses are degenerated into circles and the hyperbolae into straight lines (several examples are designated by $\mathrm{D}$ ).

c) Homeotropic smectic with planes, parallel to the glass plates (regions $\mathrm{E}$ ).

The nucleation of different types of focal conics clearly shows that the formation of the $\operatorname{SmA}$ phase starts simultaneously in the whole liquid crystal layer. This is due to the reduced N-SmA phase transition temperature $T_{\mathrm{c}}$ resulting from the initial deformations in the nematic phase [35], [56], [57]. This was confirmed in our experiment by the existence of pronounced transition N-SmA stripes (first-order phase transition) and by the strong dependence of the N-SmA phase transition temperature, $T_{\mathrm{c}}$, on the strength of the applied voltage, and on the degree of the bend-splay deformations in the nematic phase.

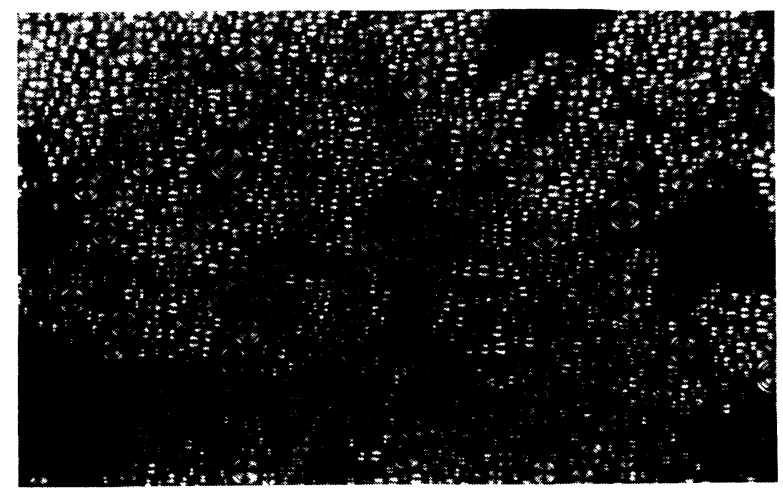

Fig. 6. - Intermediate structures, designated by $\mathrm{F}$, in planar CBOOA $\left(26 \mu \mathrm{m}\right.$ thick, $U=10 \mathrm{~V}, n_{0} / / \mathrm{P} \perp \mathrm{A}$, cooling rate $15^{\circ} \mathrm{C} / \mathrm{min}$.). One can observe circular-line focal conics designated by $\mathrm{D}$ and homeotropic $\mathrm{SmA}$ regions with planes parallel to the glass plates, designed by $\mathrm{E}$. The short side of the photo corresponds to $215 \mu \mathrm{m}$ (magnif. $\times 320$ ).

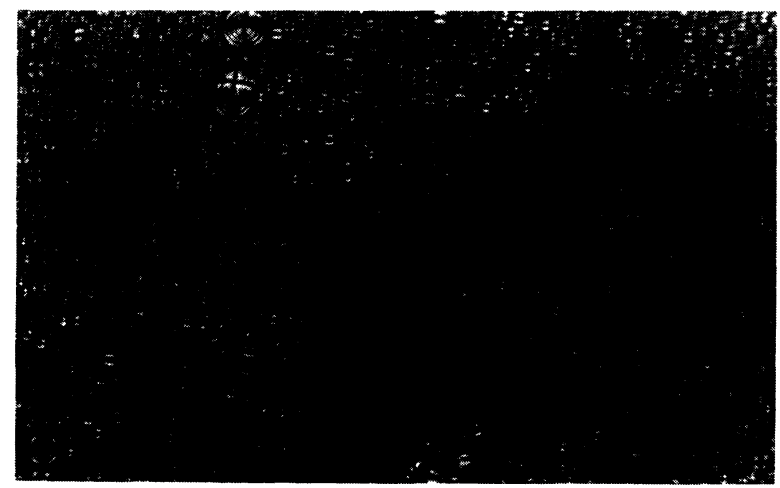

Fig. 7. - Intermediate structures designated by $F$ in planar CBOOA $\left(26 \mu \mathrm{m}\right.$ thick, $U=10 \mathrm{~V}, n_{0} / / \mathrm{P} \perp \mathrm{A}$, cooling rate $15^{\circ} \mathrm{C} / \mathrm{min}$.). One can observe many circular-line focal conics designated by $\mathrm{D}$. The short side of the photo corresponds to $215 \mu \mathrm{m}$ (magnif. $\times$ 320).

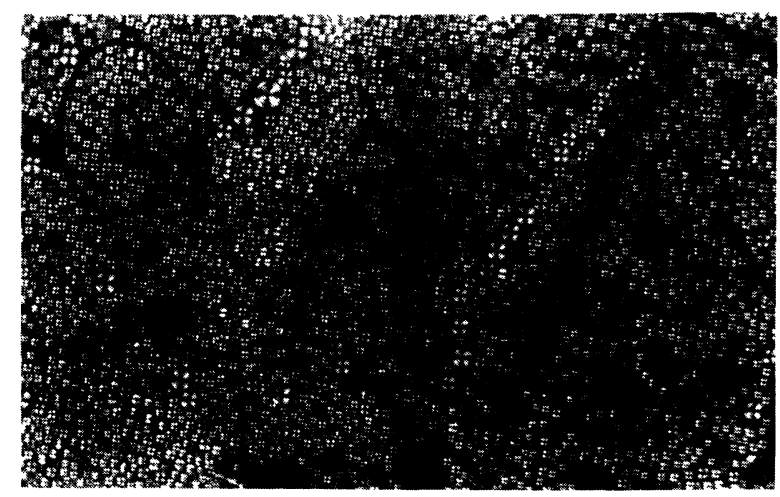

Fig. 8. - Circular-line focal conics designated by $\mathrm{D}$ in planar CBOOA $\left(26 \mu \mathrm{m}\right.$ thick, $U=15 \mathrm{~V}, n_{0} / / \mathrm{P} \perp \mathrm{A}$, cooling rate $15^{\circ} \mathrm{C} / \mathrm{min}$.). These focal conics are in various planes parallel to the glass plates. The short side of the photo corresponds to $215 \mu \mathrm{m}$ (magnif. $\times 320$ ). 
Further increase of the applied voltage

$$
\left(3 U_{\text {th }}<U<10 U_{\text {th }}, U_{\text {th }}=1.1 \mathrm{~V}\right)
$$

(Figs. 6-8) the following textures appeared as the temperature was decreased : bend-splay nematic, stripe-like textures, intermediate texture (designated in Fig. 7 and Fig. 6 by F), confocal textures in which the ellipse was degenerated into a circle and the hyperbola into a straight line. The rest of the liquid crystal is a homeotropic smectic with smectic planes parallel to the glass plates (Fig. 9) (as mentioned in the introduction the same picture has been proposed by Friedel [47] to explain a possible orientation of the liquid crystal molecules at the free surface of a SmA drop placed on a supporting glass plate). The circular focal conic textures were formed from the stripes through one intermediate structure ( $F$ in Figs. 6 and 7). Sometimes the focal conic grew from the centre of this structure as one can see from figures 6 and 7 (this type of growth of the circular focal conic domains is designated by $\mathrm{G}$ ). The exact distribution of the smectic layers in these intermediate structures (seen as bright bands in crossed nicols) is not clear. These structures arise only as a consequence of the relaxation which takes place between transitional stripes and the circular-line confocal domains which are energetically stable. One possible explanation is the formation of different types of grain boundaries which relax to the stable and energetically more favourable circular confocal domains. These intermediate textures also form a connecting link between the transitional stripes and the general Dupin cyclides (see Figs. 4 and 5).

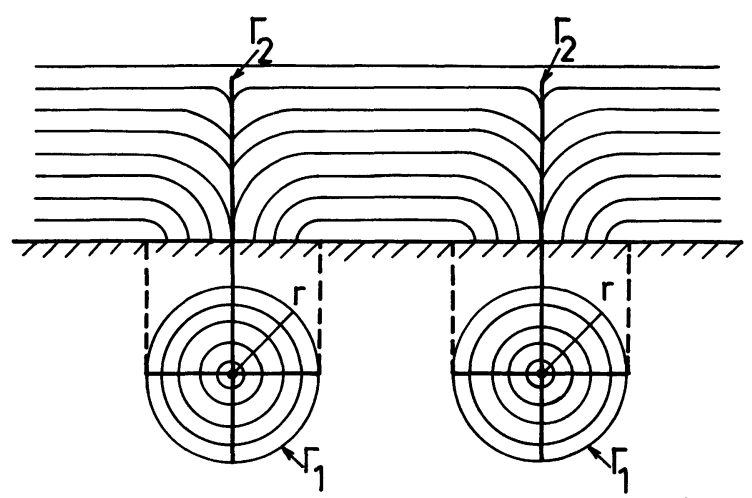

Fig. 9. - Schematic representation of the SmA phase obtained from the strongly deformed nematic phase. The molecules are perpendicular to the smectic layers.

In a strongly deformed nematic, the $\operatorname{SmA}$ phase starts to nucleate in the middle of the liquid crystal layer where the nematic molecules are oriented normal to planes, parallel to the electrode surfaces and the transition temperature $T_{\mathrm{c} 1}$ is higher than that in the boundary regions, $T_{\mathrm{c} 2}\left(T_{\mathrm{c} 1}>T_{\mathrm{c} 2}\right)$. Adjustment between the molecular orientations at the surfaces and those in the bulk is achieved with circular-line confocal structures built in homeotropic SmA liquid crystals with planes parallel to the glass plates.

At very high voltages $\left(30 U_{\text {th }}<U<40 U_{\text {th }}\right.$, $\left.U_{\mathrm{th}}=1.1 \mathrm{~V}\right)$ applied to the liquid crystal in the nematic phase we observed dark regions in crossed nicols which correspond to a homeotropic $\operatorname{SmA}$ single liquid crystal with a few isolated small circular focal conics (Fig. 10).

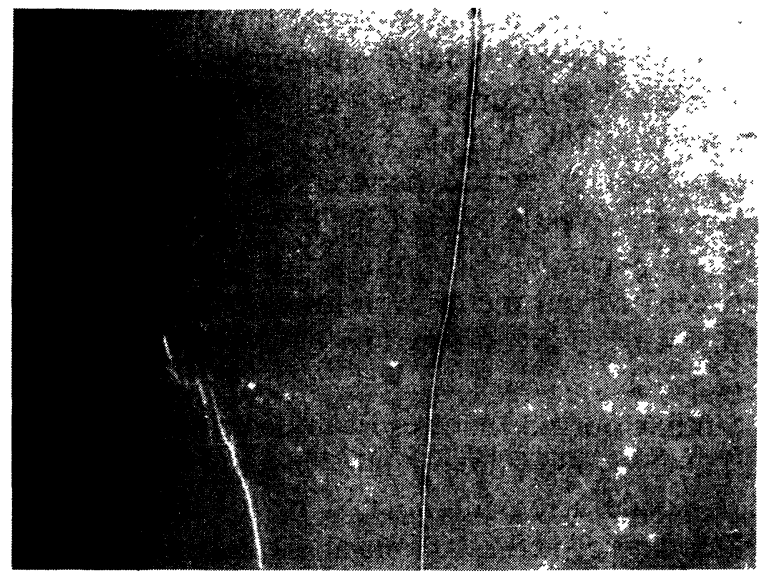

Fig. 10. - Homeotropic SmA liquid crystal with planes parallel to the glass plates in planar CBOOA $(26 \mu \mathrm{m}$ thick, $U=30 \mathrm{~V}$, $n_{0} / / \mathrm{P} \perp \mathrm{A}$, cooling rate $15^{\circ} \mathrm{C} / \mathrm{min}$.). One can observe isolated circular-line focal conics. The short side of the photo corresponds to $215 \mu \mathrm{m}$ (magnif. $\times 320$ ).

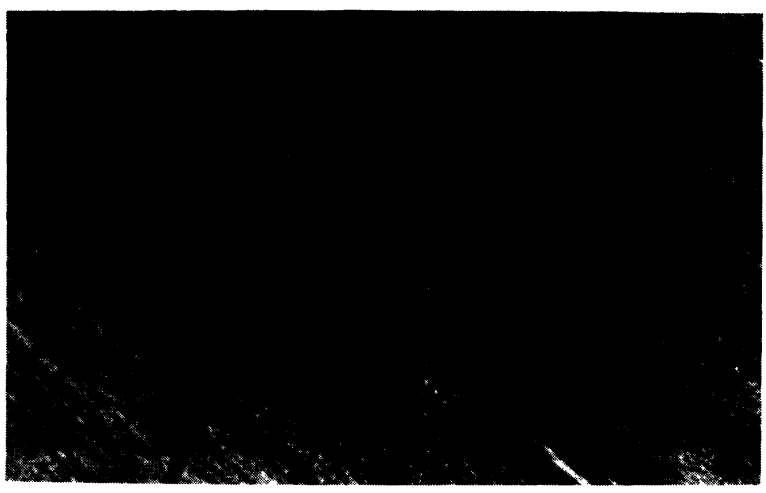

Fig. 11. - Smectic structures in crystal phase of CBOOA $(26 \mu \mathrm{m}$ thick, $U=10 \mathrm{~V}, \mathrm{P} \perp \mathrm{A}$, cooling rate $15^{\circ} \mathrm{C} / \mathrm{min}$.). The short side of the photo corresponds to $215 \mu \mathrm{m}$ (magnif. $\times 320$ ).

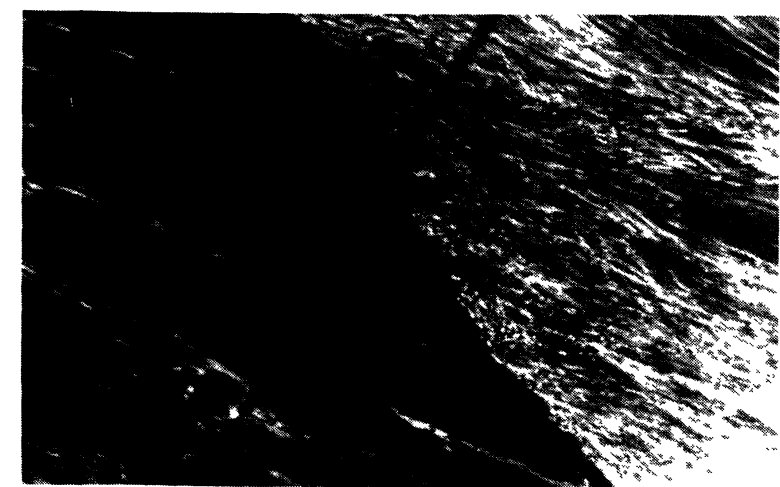

Fig. 12. - Smectic structures in the crystal phase of $\mathrm{CBOOA}$ ( $26 \mu \mathrm{m}$ thick, $U=5 \mathrm{~V}, \mathrm{~A} \perp \mathrm{P}$, cooling rate $15^{\circ} \mathrm{C} / \mathrm{min}$.). The short side of the photo corresponds to $215 \mu \mathrm{m}$ (magnif. $\times 320$ ). 
A curious experimental fact was observed after cooling the layers from the SmA phase to the crystalline phase : frequently confocal domains or only the splay orientation in the circles remain on the crystal lattice (Figs. 11 and 12).

3. General discussion for the patterns in figures 3-10. - All photographs were taken with natural light between crossed nicols. We note that this position of the nicols is more favourable for optical investigations of the SmA structures observed. The concentric rings observed in the domains result from changes in the total phase difference between ordinary and extraordinary waves (as noted by Perez and Proust [43]) and are seen only for splay molecular orientations between crossed nicols even for nematics in the form of droplets, as investigated by Cladis [58] and Candau et al. [59].

Similar optical pictures (well described by Niessen and Onden [60]) were obtained in a thick homeotropic SmA single layer, illuminated by a convergent light beam. These pictures confirm the types of the SmA textures observed. In connection with these pictures it is interesting to note their local optical biaxiality, which has been investigated by Galerne [61] in oriented defects in samples of lamellar liquid crystals.

The circular shape of the confocal domains may also be observed without nicols in natural light (see Fig. 5 in Ref. [43]). These shapes are clearly distinguished from the polygon shaped picture obtained under the same optical conditions (see Fig. 3 in Ref. [43]).

The circular-line confocal domains appear in parallel nicols as elongated spots analogous to those obtained by Ribotta [62] in the SmA phase of the liquid crystal BBAA.

In conclusion, favourable conditions for observing the confocal domains generated in the boundary regions are the use of natural light, crossed nicols and high magnification $(\times 400)$.

The formation of differently distributed confocal domains is more easily obtained by cooling from deformed nematic layer than by using special means for mechanical undulations of the SmA layers [63]-[66].

4. Competition between wall alignment and electric field alignment (in the nematic phase) in a SmA. A number of workers have considered possibilities for the matching of the surface orientation with the bulk orientation of the liquid crystal molecules, including grain boundaries [36], [37], [40], [67], or systems of focal conics [36], [37] filling the available space by Appolonian packing (Fig. 13).

It is interesting to note that in our case the liquid crystal behaviour follows closely that suggested by Bouligand [37] : in his model the ellipses are all circles and are in the plane of the surface ; the hyperbolae are straight lines normal to the surface. The filled region

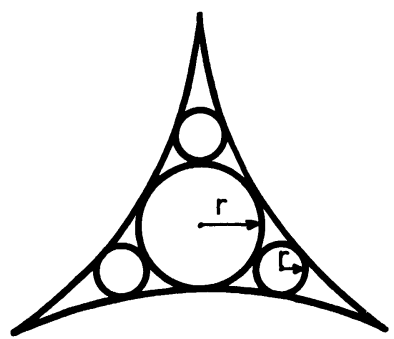

Fig. 13. - Appolonian packing of the confocal domains.

associated with one pair of conics is a cylinder of revolution based on the circle. These cylinders fill all the available space by Appolonian packing. No polygons are required.

However, our experiments differ in two ways from Bouligand's model : in rare cases the filling is Appolonian, in many cases random and stops for confocal domains having a critical circle radius which depends on the strength of the surface coupling. Further filling of the space occurs by homeotropic SmA with planes parallel to the glass plates i.e. in this case the surface anchoring is insufficient for the large bending of the smectic layers.

It is important to note that these domains were observed in a wide range of temperature gradients : between $15^{\circ} \mathrm{C} / \mathrm{min}$. (using a cell to store the thermal energy) and $0.1{ }^{\circ} \mathrm{C} / \mathrm{min}$. using a Mettler type hot stage. The large temperature gradients were in the plane of the electrodes where the confocal domains had a circular shape. These observations suggest that the formation of the confocal domains is due to a balance between the total surface energy on the area confined within the circles and the total elastic energy of the focal conic textures.

The domain radius was usually in the range of 1.3-2.6 $\mu \mathrm{m}$. Sometimes at lower voltages the radius of the circular line domains was one half of the thickness of the liquid crystal layer (Figs. 4-5) which indicates that the formation of the confocal domains in these cases was due to non-equilibrium growth effects rather than to an energy balance. At higher voltages (Figs. 6-8) the size of the confocal domains varies by a factor of 2 and is related to the formation of the SmA phase only in the surface regions. In some cases the circular confocal domains obtained were at different levels with respect to the boundary planes (the barely visible crosses in Fig. 8) due to growth effects. Another more unlikely explanation could be their formation at the lower glass plate.

The liquid crystal appears to be a single smectic A for values of the applied voltage above $30 \mathrm{~V}$ in agreement with the light microscope observations.

5. Estimation of the surface energy in the nematic and smectic phases. - The smectic phase arises from a strongly deformed nematic region. The distribution of the domains of different size is related to surface anchoring in the nematic and smectic phases. 
We suggest that the dark regions where the SmA phase is homeotropic correspond to a smaller surface energy of the two phases : $\mathbf{N}$ and SmA. I.e. these pictures are a special distribution of the surface energy : the confocal domains with larger radius correspond to regions with smaller surface energy.

The alignment of smectics on $\mathrm{SiO}$ treated substrates under vacuum evaporation has been mainly investigated in the bulk of the liquid crystal (see e.g. [68]). In the literature there are no estimates of the size of the surface energy of SmA liquid crystal molecules. However, this is important both for experimental investigations and for practical applications of the SmA liquid crystals.

a) Estimates of $\theta$-SmA surface energy [69]. The $\theta-\mathrm{SmA}$ surface energy can be estimated from theoretical results obtained by Kléman [70] for the energy of these circular-line confocal domains :

$$
W_{\text {cd }}=2 \pi K_{1} r_{\mathrm{c}}\left(\left(\ln 2 r_{\mathrm{c}} / r_{\mathrm{cor}}\right)-2\right)
$$

where $r_{\mathrm{c}}$ is the radius of the circle, $r_{\text {cor }}$ is the coreradius and $K_{1}$ is the splay elastic coefficient.

The energy of the molecules in the surface regions is maximized and takes the value, $W_{\mathrm{s}}(\pi / 2)$, when the smectic layers are aligned and parallel to the boundaries. This total energy is equal to the sum of one half of the energy of the confocal domains just above their disappearance,

$$
W_{\mathrm{cd}}=\pi K_{1} r_{\mathrm{c}}\left(\left(\ln 2 r_{\mathrm{c}} / r_{\mathrm{cor}}\right)-2\right)
$$

and the energy of the core line,

$$
W_{\mathrm{cl}} \approx 2 E_{\mathrm{cor}} r_{\mathrm{c}} \text {. }
$$

The energy of the core line per unit length is of the order of $K_{1}$ [37]. The core radius is of the order of several smectic layer thicknesses $\sim 100 \AA$. We calculate the mean value of the $\theta$-surface energy of the SmA liquid crystal for glass plates treated with $\mathrm{SiO}$ is,

$$
W_{\mathrm{s}} \approx 4 \times 10^{-2} \mathrm{erg} / \mathrm{cm}^{2}
$$

from the relation,

$$
W_{\mathrm{s}}=\left(K_{1} / r_{\mathrm{c}}\right)\left(\left(\ln 2 r_{\mathrm{c}} / r_{\mathrm{cor}}\right)-2\right)+(2 / \pi)\left(K_{1} / r_{\mathrm{c}}\right)
$$

using a low value for $r_{\mathrm{c}} \sim 1.3 \mu \mathrm{m}$.

b) Estimates for $\varphi$-SmA surface energy. - The $\varphi$-liquid crystal orientation of the SmA molecules (i.e. in the electrode planes) is degenerated above the $\mathrm{N}-\mathrm{SmA}$ phase transition temperature. As mentioned above, we observe the general case of Dupin cyclides in the section parallel to the hyperbola plane (see also [71]) and in the section parallel to the ellipse plane. The former were randomly distributed. The latter, due to the finite $\varphi$-surface anchoring energy, were oriented along the major axis of the ellipse, parallel to the easy axis of the SmA liquid crystal molecules which is determined by the vacuum evaporation of the $\mathrm{SiO}$ thin films. (Note that there were thermal gradients perpendicular to the easy axis of the SmA liquid crystal molecules.) The $\varphi$-surface energy should be calculated as the difference between the energy of the general Dupin cyclide (see the theoretical result obtained by Kléman [69]) and the energy of the circular-line domain obtained after relaxation of the Dupin cyclide at lower temperatures. This, however, was not performed in our experiment.

The determination of $\theta$ - and $\varphi$-surface energy is more complex for cases where the filling of space is achieved with systems of polygons [43], [73] (the smallest confocal domains are replaced by dislocations of rotation [39], [74]). It is clear that the magnitude of the surface energy (which is probably very high) may be obtained after the transformation : systems of polygons $\rightarrow$ homeotropic SmA [43] if the total elastic energy of the polygons per unit volume is known. This, however, is a very difficult problem.

c) Estimates for $\theta$-surface energy in the nematic phase. - Application of high electric fields in the nematic phase should completely orient the liquid crystal molecules along the electric field lines (the dielectric anisotropy of CBOOA investigated in the nematic phase is positive) when the surface anchoring is finite. This is difficult to confirm for liquid crystals showing only a nematic phase although there are sensitive optical methods such as total internal reflection, etc. This, however, is easy to confirm for liquid crystals having two or more liquid crystal phases. As the coherence length in the boundary regions, consisting of splay-bend deformations in the nematic phase (see de Gennes [75] and [72]) tends to zero when cooling, the transitional stripes characteristic for first-order or slightly first-order N-SmA phase transitions disappear and one can obtain a single SmA liquid crystal [44], [45], [76]. The value of the surface coupling parameter in the nematic phase $W_{\mathrm{sN}}$ can then be calculated from the balance between the negative maximal electric energy per $\mathrm{cm}^{2}$ of the liquid crystal and the positive maximal surface energy :

$$
(|\Delta \varepsilon| / 8 \pi) E^{2} d=W_{\mathrm{sN}}(\pi / 2) .
$$

For an applied voltage with a strength of $30 \mathrm{~V}$ (Fig. 10) $\Delta \varepsilon \simeq 5.5$ [72] and $d \simeq 26 \mu \mathrm{m}$ we calculate the following value of the surface coupling parameter $W_{\mathrm{sN}}$ in the nematic phase :

$$
W_{\mathrm{sN}} \approx 0.85 \mathrm{erg} / \mathrm{cm}^{2} .
$$

The ratio of this surface energy with that obtained for interaction of the smectic A molecules with the same boundaries, $W_{\mathrm{s}}: W_{\mathrm{s}} \approx 4 \times 10^{-2} \mathrm{erg} / \mathrm{cm}^{2}$, is about 20. We suggest this large difference is due chiefly to physico-chemical short-range molecular interactions in both phases : nematic and $\operatorname{SmA}$ and to a lower extent to surface relief effects. 
6. Influence of the electric field in the SmA phase. All textures in the SmA phase remain unchanged after removal of the electric field. This is due to dielectric anisotropy being positive in both nematic and SmA phases. Consider the following possibilities for storage information :

a) the generated focal conic textures contain storage information about the surface energy as expressed by different values of the confocal radius;

b) the scattering textures due to the presence of confocal domains containing disclinations in their structure should have a long life [75]. This was confirmed by storage of part of these domains in the crystal lattices (Figs. 11 and 12).

Goscianski et al. [40] consider the possibility of grain-boundary generation resulting from competition between surface anchoring and the bulk electric deforming torques for liquid crystals 40.8 with negative dielectric anisotropy.

Hareng et al. [77] observe focal conics induced by an electric field in an initially homeotropic COB sample.

Of interest is the investigation of the growing processes of SmA phase from strongly deformed surface regions in the nematic phase when the sign of the dielectric anisotropy in both nematic and SmA phases is different or with homeotropic CBOOA in a transverse electric or magnetic field. This will be the subject of another paper.

7. Conclusions. - The SmA phase was obtained after cooling from a strongly deformed planar nematic liquid crystal CBOOA. At low voltages

$$
1.5 U_{\text {th }} \leqslant U \leqslant 3 U_{\text {th }}
$$

( $U_{\mathrm{th}}=1.1 \mathrm{~V}$ is the threshold voltage) the textures obtained were in agreement with Cladis and Torza's experimental results for hybrid deformed layers. At higher voltages the SmA phase was first formed in the middle of the liquid crystal cell. In the boundary regions a system of confocal domains was observed in which the ellipse is degenerated into a circle and the hyperbola into a straight line. The remaining part of the smectic liquid crystal is homeotropic with layers parallel to the boundaries. The filling process which is more random than Appolonian, ends with the formation of circular confocal domains having some critical radius which corresponds to a maximum value of the surface energy. The smectic liquid crystal is then homeotropic. The surface energies in the SmA phase and in the nematic phase have also been calculated.

Although all kinds of focal-conic domains were obtained adjustment between the surface orientation and the bulk orientation is not exhausted with these structures. We have considered only those pictures which are both typical and of some use for the determination of the surface energies which are important both for experimental research and for technical application of the nematic and SmA liquid crystals.

Acknowledgments. - The experimental results were obtained during the author's stay in the Laboratoire de Physique des Solides, Orsay. $\mathrm{He}$ is indebted to Prof. E. Guyon for making possible the visit to the University of Paris. He thanks Profs. M. Kléman and G. Durand, and Drs. Ribotta, Galerne, Prost, Martinet, Strzelecki, Rault and Leshek for discussing several problems. The author is also indebted to M. Boix for the preparation of the glass plates treated with thin layers of $\mathrm{SiO}$.

\section{References}

[1] De Gennes, P. G., Solid State Commun. 10 (1972) 753.

[2] McMillan, W. L., Phys. Rev. A 6 (1972) 936.

[3] Brochard, F., J. Physique 34 (1973) 411.

[4] JAHNig, F. and Brochard, F., J. Physique 35 (1974) 301.

[5] Halperin, B. I., Lubensky, T. C. and Ma, S., Phys. Rev. Lett. 32 (1974) 292

[6] Halperin, B. I. and Lubensky, T. C., Solid State Commun. 14 (1974) 997.

[7] Chen, Jing-Huei and Lubensky, T. C., Phys. Rev. A 14 (1976) 1202.

[8] McMillan, W. L., Phys. Rev. A 9 (1974) 1720.

[9] Martins, A. F., Diogo, A. C. and Vaz, N. P., Ann. Phys. (1978) 361.

[10] Hossain, Kh. A., Swift, J., Chen, Jing-Huei and LuBENSKY, T. C., Phys. Rev. B 19 (1979) 432.

[11] McMillan, W. L., Phys. Rev. A 7 (1973) 1419.

[12] McMillan, W. L., Phys. Rev. A 7 (1973) 1673.

[13] Litster, J. D., Als-Nielsen, Birgeneau, R. J., Dana, S. S., Davidov, D., Garcia-Go Lding, F., Kaplan, M., Sainya, C. R. and Schaeting, R. J., J. Physique Colloq 40 (1979) C3-339.
[14] ConRad, H. M., Stiller, H. H., FrischKoRN, C. G. B. and ShIRANe, G., Solid State Commun. 23 (1977) 571.

[15] Delay, M., Ribotta, R. and Durand, G., Phys. Rev. Lett. 31 (1973) 443.

[16] Salin, D., Smith, I. W. and Durand, G., J. Physique Lett. 34 (1973) L-443.

[17] Salin, D., Thèse de 3e Cycle, Paris (1974).

[18] Chu, K. C. and McMillan, W. L., Phys. Rev. A 11 (1975) 1059.

[19] BiReCKi, H., Schaetzing, R., Rondelez, F. and Litster, J. D., Phys. Rev. Lett. 36 (1976) 1376.

[20] BireCKI, H. and Litster, J. D., Mol. Cryst. Liq. Cryst. 42 (1977) 33.

[21] Delaye, M., Thesis, Paris (1974).

[22] Wise, R. A., Olah, A. and Doane, J. W., J. Physique Colloq. 36 (1975) C1-117.

[23] BACRI, J. C., J. Physique Colloq. 36 (1975) C1-123.

[24] Cheung, L., Meyer, R. B. and Gruler, H., Phys. Rev. Lett. 31 (1973) 349.

[25] Cladis, P. E., Phys. Rev. Lett. 31 (1973) 1200.

[26] Huang, Cheng-Chei, Pindak, R. S., Flanders, Ph. J. and Ho, J. T., Phys. Rev. Lett. 33 (1974) 400. 
[27] D’Humières, D. and Leger, L., J. Physique Colloq. 36 (1975) C1-113.

[28] SaKamoto, A., Yoshino, K., Kubo, U. and Inuishi, Y., Japan. J. Appl. Phys. 15 (1976) 745.

[29] Hardouin, F., Achard, M. F., Sigaud, G. and Gasparoux, H., Phys. Lett. 49A (1974) 25.

[30] Hardouin, 'F., Achard, F. and Gasparoux, H., Solid State Commun. 14 (1974) 453.

[31] AChard, M. F. and Sigaud, G., J. Physique Colloq. 36 (1975) C1-107.

[32] Kim, M. G., Park, S., CoOper, Sr. M. and Letcher, S. V., Mol. Cryst. Liq. Cryst. 36 (1976) 143.

[33] Williams-Dorlet, C., Thèse Doct. Sci. Phys. (1976).

[34] Marignan, J., Malet, G. and Parodi, O., Ann. Phys. 3 (1978) 221.

[35] Cladis, P. E. and Torza, S., J. Appl. Phys. 46 (1975) 584.

[36] Parodi, O., Solid State Commun. 11 (1972) 1503.

[37] Bidaux, R., Boccara, N., Sarma, G., de Seze, L., de Gennes, P. G. and Parodi, O., J. Physique 34 (1973) 661.

[38] Williams, C. and Kléman, M., J. Physique Colloq. 36 (1975) C1-315.

[39] Kléman, M., Advances in LCs, vol. 1 (1975) 267.

[40] Goscianski, M., Leger, L. and Mircea-Roussel, A., J. Physique Lett. 36 (1975) L-313.

[41] Rault, J., Philos. Mag. 34 (1976) 753.

[42] Rosenblatt, Ch. S., Pindak, R., Clark, N. A. and Meyer, R. B., J. Physique 38 (1977) 1105.

[43] Perez, E. and Proust, J. E., J. Physique Lett. 38 (1977) L-117.

[44] LE BERRE, S. and HARENG, M. : Etudes des phénomènes basse fréquence dans les Sm A, Thomson-CSF (1977) 1-48.

[45] Le Berre, S. and Hareng, M., Ann. Phys. 3 (1978) 327.

[46] The author indebted to Prof. M. Kléman who participated in the discussion.

[47] Kléman, M., Points. Lignes. Parois (Les Editions de Physique) 1979, tome I, p. 142.

[48] Rajan, V. S. V. and Picot, J. J. C., Mol. Cryst. Liq. Cryst. 20 (1973) 55.

[49] Yun, C. K., Picot, J. J. C. and Fredrickson, A. G., J. Appl. Phys. 42 (1971) 4764.

[50] Rajan, V. S. V. and Picot, J. J. C., Mol. Cryst. Liq. Cryst. 17 (1972) 109.

[51] Urback, W., Hervet, H. and Rondelez F., Mol. Cryst. Liq. Cryst. 46 (1978) 209.

[52] Rondelez, F., Urback, W. and Hervet, H., Phys. Rev. Lett. 41 (1978) 1058.
[53] Friedel, G., Ann. Phys. 18 (1922) 273.

[54] Bouligand, Y., J. Physique 33 (1972) 525.

[55] Demus, D. and Richter, L., Textures of Liquid Crystals (VEB Deutscher Verlag für Grundstoffindustrie, Leipzig) 1978 , p. 67

[56] SaKamoto, A., Yoshino, K., Kubo, U. and InUishi, Y., Japan. J. Appl. Phys. 15 (1976) 545.

[57] Sakamoto, A., Yoshino, K., Kubo, U. and Inuishi, Y., Japan. J. Appl. Phys. 15 (1976) 745.

[58] KLéMan, M., Bull. Soc. Fr. Miner. Cristallogr. (1972) 95, 215230.

[59] Candau, S., Le Roy, P. and Debeauvais, F., Mol. Cryst. Liq. Cryst. 23 (1973) 283.

[60] Niessen, A. K. and Den Onden, A., Philos. Res. Rep. 29 (1974) 119.

[61] Galerne, Y., J. Physique Lett. 40 (1979) L-73.

[62] Ribotta, R., Thèse doct. sci., Paris (1975) 87.

[63] RiBotta, R., J. Physique Colloq. 37 (1976) C1-7.

[64] Ribotta, R., J. Physique Colloq. 37 (1976) C3-149.

[65] Ribotta, R. and Durand, G., J. Physique 38 (1977) 179.

[66] Adomenas, P. V., Vistin', L. K., Mesropyan, A. N., Tubelite, A. A., Chistyakov, I. G., Chumakova, S. P., EigenBRot, V. M. and Yakovenko, S. S., Sov. Phys. JETP 46 (1977) 999, Zh. Eksp. Teor. Phys. 73 (1977) 1903.

[67] Chuvyrov, A. N. and Kuvatov, Z., Kristallografiya 18 (1973) 344.

[68] Urback, W., Borx, M., Guyon, E., Appl. Phys. Lett. 25 (1974) 479.

[69] These results have been presented for a patent application, No. 48390 of 4.7.1980.

[70] KlÉMAN, M., J. Physique 38 (1977) 1511

[71] Friedel, J., J. Physique Colloq. 40 (1979) C3-45.

[72] Cohen, M., Pieranski, P., Guyon, E. and Mitesku, C. D., Mol. Cryst. Liq. Cryst. 38 (1977) 97.

[73] Proust, J. E. and Perez, E., J. Physique Lett. 38 (1977) L-91.

[74] KlÉman, M., Philos. Mag. 34 (1976) 79.

[75] De Gennes, P. G., The Physics of Liquid Crystals (Clarendon Press, Oxford) 1974.

[76] Hataevich, V. I. and Zheinaly, A. Kh., Pis'ma Zh. T. Phys. 5 (1979) 651.

[77] Hareng, M., Le Berre, S. and Metzger, J. J., Appl. Phys. Lett. 27 (1975) 575. 